\title{
DEVELOPMENT OF SINGULARITIES IN SOLUTIONS OF THE EQUATIONS OF NONLINEAR THERMOELASTICITY*
}

\author{
BY \\ C. M. DAFERMOS ${ }^{1}$ (Lefschetz Center for Dynamical Systems, Brown University) \\ AND \\ L. HSIAO (Institute of Mathematics, Academia Sinica, People's Republic of China)
}

1. Introduction. The Lagrangian formulation of the equations of motion of a one-dimensional, homogeneous medium of unit reference density reads

$$
\left\{\begin{array}{l}
\partial_{t} u-\partial_{x} v=0 \\
\partial_{t} v-\partial_{x} \sigma=0
\end{array}\right.
$$

where $u$ denotes deformation gradient (i.e., the inverse of density), $v$ is velocity, and $\sigma$ is the Piola-Kirchhoff stress. When the material is elastic, the stress is determined by the deformation gradient through a constitutive relation

$$
\sigma=p(u), \quad 0<u<\infty,
$$

where $p(u)$ is strictly increasing,

$$
p_{u}(u)>0, \quad 0<u<\infty,
$$

so that the system (1.1) is strictly hyperbolic.

The destabilizing effect of nonlinear elastic response may be seen by studying the propagation of acceleration waves, that is, jump discontinuities in the acceleration $\partial_{t} v$ (as well as other first partial derivatives of $u$ and $v$ ). Acceleration waves propagate along characteristics with speed

$$
V(t)= \pm p_{u}^{1 / 2}(u)
$$

When the elastic medium ahead of the wave front is at rest, i.e., $u=\bar{u}=$ constant, $v=0$, then the speed $V$ of propagation is constant, equal to $\pm p_{u}^{1 / 2}(\bar{u})$, and the amplitude, measured by the jump of acceleration across the wave, satisfies the ordinary differential equation

$$
\dot{\alpha}+\left(p_{u u}(\bar{u}) / 2 V^{3}\right) \alpha^{2}=0 .
$$

\footnotetext{
* Received March 29, 1985.

${ }^{1}$ Supported by the National Science Foundation through Grant \#DMS-8205355, by the U. S. Army under Contract \# DAAG-29-83-K-0029 and by the Office of Naval Research under Contract \# N00014-83-K-0542.
} 
Therefore, if $p_{u u}(\bar{u}) \neq 0$ and $\alpha(0) p_{u u}(\bar{u})<0$, then the amplitude explodes in a finite time and, presumably, the acceleration wave breaks into a shock wave.

The above analysis suggests that solutions to the Cauchy problem for (1.1), (1.2), with smooth initial data

$$
u(x, 0)=u_{0}(x), \quad v(x, 0)=v_{0}(x), \quad-\infty<x<\infty,
$$

may develop discontinuities in a finite time. This was indeed established by the following argument of Lax [10]. Consider the Riemann invariants, defined by

$$
r=v+\int_{\bar{u}}^{u} p_{u}^{1 / 2}(\xi) d \xi, \quad s=v-\int_{\bar{u}}^{u} p_{u}^{1 / 2}(\xi) d \xi,
$$

and apply the operators of differentiation along the characteristic directions, viz.,

$$
=\partial_{t}-p_{u}^{1 / 2}(u) \partial_{x}, \quad '=\partial_{t}+p_{u}^{1 / 2}(u) \partial_{x} .
$$

A simple calculation using (1.1), (1.2) yields

$$
\grave{r}=0, \quad \dot{s}=0,
$$

i.e., $r$ stays constant along backward characteristics and $s$ stays constant along forward characteristics. Consequently, so long as a classical solution of (1.1), (1.2), (1.6) exists, $r, s$, and thereby also $u, v$, will remain uniformly bounded by constants that depend solely on the $L^{\infty}$ norm of the initial data $u_{0}(\cdot)$ and $v_{0}(\cdot)$. Next consider the evolution, along characteristics, of partial derivatives of the Riemann invariants,

$$
w=\partial_{x} r, \quad \omega=\partial_{x} s .
$$

A lengthy but interesting calculation [10] yields

$$
\left\{\begin{array}{l}
{\left[p_{u}^{1 / 4}(u) w\right]-\frac{1}{4} p_{u}^{-5 / 4}(u) p_{u u}(u)\left[p_{u}^{1 / 4}(u) w\right]^{2}=0,} \\
{\left[p_{u}^{1 / 4}(u) \omega\right]^{\prime}-\frac{1}{4} p_{u}^{-5 / 4}(u) p_{u u}(u)\left[p_{u}^{1 / 4}(u) \omega\right]^{2}=0 .}
\end{array}\right.
$$

It follows that if $p_{u u}(u)$ does not change sign, then $w, \omega$, and thereby also the first partial derivatives of $u, v$, will generally blow up in finite time, i.e., in general the initial-value problem (1.1), (1.2), (1.6) does not have a globally defined classical solution. Solutions generally blow up even when $p_{u u}(u)$ changes sign (cf. Klainerman and Majda [8]), but the proof is quite technical.

It is interesting to discuss the situation in which the destabilizing action of nonlinear elastic response, exhibited above, coexists and competes with the damping action of dissipation. For general background information see, e.g., [3]. The prevailing conjecture is that dissipation manages to restrain waves of small amplitude but is incapable of preventing the breaking of waves with large amplitude. The simplest example of this type arises when dissipation is induced by frictional damping. This case can be treated by mimicking the analysis for (1.1), outlined above (cf. Nishida [13]). In recent years, considerable effort has been expended (cf., e.g. $[11,5,7,6,4])$ in the study of stabilizing effects of dissipation induced by memory response (viscosity of the Boltzmann type).

Here we investigate the effectiveness of damping induced by heat diffusion, within the framework of one-dimensional thermoelasticity. The Lagrangian formulation of the balance equations of mass, momentum, and energy for one-dimensional homogeneous media 
with unit reference density reads

$$
\left\{\begin{array}{l}
\partial_{t} u-\partial_{x} v=0 \\
\partial_{t} v-\partial_{x} \sigma=0 \\
\partial_{t}\left(\varepsilon+\frac{1}{2} v^{2}\right)-\partial_{x}(\sigma v)=\partial_{x} q
\end{array}\right.
$$

where, as above, $u$ is the deformation gradient, $v$ is velocity, and $\sigma$ is stress, while $\varepsilon$ denotes the internal energy and $q$ denotes the heat flux. In a thermoelastic material, stress, internal energy, and heat flux are determined by the constitutive relations

$$
\varepsilon=\hat{\varepsilon}(u, T), \quad \sigma=\hat{\sigma}(u, T), \quad q=\hat{q}\left(u, T, \partial_{x} T\right),
$$

where $T$ stands for absolute temperature. The functions $\hat{\varepsilon}$ and $\hat{\sigma}$ must satisfy the compatibility relation

$$
\hat{\varepsilon}_{u}(u, T)=\hat{\sigma}(u, T)-T \hat{\sigma}_{T}(u, T),
$$

which is induced by the Second Law of Thermodynamics. Moreover, $\hat{q}$ should be an increasing function of $\partial_{x} T$ that vanishes when $\partial_{x} T=0$, and the specific heat at fixed volume should be positive, i.e.,

$$
\hat{q}(u, T, 0)=0, \quad \hat{q}_{h}(u, T, h)>0, \quad \hat{\varepsilon}_{T}(u, T)>0 .
$$

The propagation of acceleration waves in thermoelastic media is studied in [2] in great detail and generality. A brief discussion that would suffice for the present purposes can be found in [3]. The speed of propagation is given by

$$
V(t)= \pm \hat{\boldsymbol{\sigma}}_{u}^{1 / 2}(u, T),
$$

which should be compared with (1.4). When the thermoelastic medium ahead of the wave front is at rest, i.e., $u=\bar{u}=$ constant, $v=0$, and at constant temperature $T=\bar{T}=$ constant, then the speed $V$ of propagation is constant and equal to $\pm \hat{\sigma}_{u}^{1 / 2}(\bar{u}, \bar{T})$, and the amplitude, measured, as before, by the jump of acceleration across the wave, satisfies the Bernoulli equation

$$
\dot{\alpha}+\frac{\hat{\sigma}_{u u}(\bar{u}, \bar{T})}{2 V^{3}} \alpha^{2}+\frac{\bar{T} \hat{\sigma}_{T}^{2}(\bar{u}, \bar{T})}{2 \hat{q}_{h}(\bar{u}, \bar{T}, 0)} \alpha=0,
$$

which should be compared and contrasted with (1.5). It follows that if $\hat{\sigma}_{u u}(\bar{u}, \bar{T}) \neq 0$, then the amplitude of an acceleration wave in the thermoelastic medium decays to zero exponentially fast when initially small, but may explode to infinity, in finite time, when initially large. On the strength of this observation, it is reasonable to conjecture that solutions to the system of equations of thermoelasticity exist globally in time when the initial data are smooth and "small," but they blow up in finite time when the initial data are "large." Indeed, the existence of globally defined solutions under "small" initial data was established by Slemrod [14] with the help of energy-type estimates.

The aim of our paper is to show that solutions of the equations of thermoelasticity with large initial data generally blow up in finite time. Just as in the elastic case, it turns out that the deformation gradient, velocity, and temperature stay uniformly bounded, but their first partial derivatives explode. In fact, we will consider here solutions whose range 
is contained, throughout the maximal time interval of existence, in a small neighborhood of a fixed equilibrium state $(\bar{u}, 0, \bar{T})$. With this in mind, and in order to simplify the analysis, we will assume special constitutive equations of the form

$$
\varepsilon=P(u)+c(T-\bar{T}), \quad \sigma=p(u)+\mu(T-\bar{T}), \quad q=\kappa \partial_{x} T,
$$

with $c>0, \kappa>0$, and $\mu$ constants, $p(\bar{u})=0$, which we view as approximations of (1.13) around the state $(\bar{u}, \bar{T})$. Moreover, assuming that stress varies slowly with temperature, we shall neglect ${ }^{2}$ the term $\bar{T} \hat{\sigma}_{T}$ in comparison to $\hat{\sigma}$, and so deduce from (1.14)

$$
P(u)=\int_{\bar{u}}^{u} p(\xi) d \xi .
$$

We postulate that the function $p(u)$ is smooth, satisfies (1.3), and is genuinely nonlinear in the sense

$$
p_{u u}(u)>0, \quad 0<u<\infty,
$$

so that the elastic response is destabilizing.

Let the thermoelastic body have as reference configurations the entire $x$-axis $(-\infty, \infty)$. We prescribe initial conditions

$$
u(x, 0)=u_{0}(x), \quad v(x, 0)=v_{0}(x), \quad T(x, 0)=T_{0}(x), \quad-\infty<x<\infty,
$$

where $u_{0}, v_{0}, T_{0}$ are smooth functions with range in a small neighborhood of a fixed state $(\bar{u}, 0, \bar{T})$. The main result is

Theorem 1.1. Let $u_{0}, v_{0}, T_{0}$ be $C^{2}$ functions on $(-\infty, \infty)$ such that $u_{0}-\bar{u} \in L^{2}(-\infty, \infty)$, $v_{0} \in L^{2}(-\infty, \infty), T_{0}-\bar{T} \in L^{1}(-\infty, \infty)$, and $\partial_{x} u_{0}, \partial_{x} v_{0}$, and $\partial_{x} T_{0}$ decay to zero as $|x| \rightarrow \infty$. Assume, further,

$$
\begin{gathered}
T_{0}(x)>\bar{T}, \quad-\infty<x<\infty \\
\left|\partial_{x} T_{0}(x)\right| \leqslant H, \quad-\infty<x<\infty \\
\left|u_{0}(x)-\bar{u}\right| \leqslant \delta^{1 / 2}, \quad\left|v_{0}(x)\right| \leqslant \delta^{1 / 2}, \quad\left|T_{0}(x)-\bar{T}\right| \leqslant \delta, \quad-\infty<x<\infty,\left(1 v^{2}, \quad x||^{2}+\left|T_{0}(x)-\bar{T}\right|\right\} d x \leqslant \delta \\
\int_{-\infty}^{\infty}\left\{\left|u_{0}(x)-\bar{u}\right|^{2}+\mid v_{0}(x)\right. \\
\min \left\{\partial_{x} v_{0}+p_{u}^{1 / 2}\left(u_{0}\right) \partial_{x} u_{0}\right\}+\min \left\{\partial_{x} v_{0}-p_{u}^{1 / 2}\left(u_{0}\right) \partial_{x} u_{0}\right\} \geqslant-J
\end{gathered}
$$

where $H, J$, and $\delta$ are positive constants. Given any $L>0$, there is $M>0$ which depends on $L$ as well as on the constants $\bar{u}, H, J, \delta$, such that if

$$
\max \left\{\partial_{x} v_{0}+p_{u}^{1 / 2}\left(u_{0}\right) \partial_{x} u_{0}\right\}+\max \left\{\partial_{x} v_{0}-p_{u}^{1 / 2}\left(u_{0}\right) \partial_{x} u_{0}\right\}>M,
$$

then the length of the maximal time interval of existence of any $C^{2}$ solution of (1.12), (1.18), (1.21) cannot exceed $L$. Moreover, when $\delta$ is small, the range of the solution is contained in a small neighborhood of the state $(\bar{u}, 0, \bar{T})$ throughout its maximal interval of existence.

\footnotetext{
${ }^{2}$ The constitutive relations (1.18), (1.19) conform strictly with (1.14) only when $\bar{T}=0$.
} 
In proving the above theorem, we use heavily equations analogous to (1.9), (1.11) that govern the evolution of Riemann invariants and their derivatives along characteristics. However, in contrast to the hyperbolic case, these equations now contain coupling terms that depend on $T$ and its derivatives. Therefore, it is no longer possible to establish boundedness or blow-up by restricting attention on a fixed distinct characteristic. Instead, we employ here a technique introduced in [4] that seeks to estimate solutions by monitoring the time evolution of the functions $\max _{x}|r(x, t)|, \max _{x}|s(x, t)|, \max _{x}\left|\partial_{x} r(x, t)\right|$, $\max _{x}\left|\partial_{x} s(x, t)\right|$. The proof will be presented in Sec. 2 .

The development of singularities in thermoelasticity is also discussed by McIntire [12]. McIntire considers model equations somewhat different from (2.2) and shows that a gross energy-like quantity blows up in finite time.

2. Proof of theorem. Let us assume that the initial-value problem (1.12), (1.18), (1.21) admits a $C^{2}$ solution on the strip $(-\infty, \infty) \times[0, L]$, for some positive $L \leqslant 1$. Moreover, for each $t \in[0, L], \quad u(x, t)-\bar{u} \in L^{2}(-\infty, \infty), \quad v(x, t) \in L^{2}(-\infty, \infty), \quad T(x, t)-\bar{T} \in$ $L^{1}(-\infty, \infty)$, and derivatives $\partial_{x} u, \partial_{x} v$ and $\partial_{x} T$ decay to zero as $|x| \rightarrow \infty$.

We define

$$
\theta(x, t)=T(x, t)-\bar{T} .
$$

We substitute $\varepsilon, \sigma$, and $q$ from (1.18) into (1.12), and after certain manipulations using (1.19) we arrive at the equivalent system

$$
\left\{\begin{array}{l}
\partial_{t} u-\partial_{x} v=0 \\
\partial_{t} v-p_{u}(u) \partial_{x} u-\mu \partial_{x} \theta=0 \\
\partial_{t} \theta-(\mu / c) \theta \partial_{x} v=(\kappa / c) \partial_{x}^{2} \theta
\end{array}\right.
$$

with initial conditions

$$
u(x, 0)=u_{0}(x), \quad v(x, 0)=v_{0}(x), \quad \theta(x, 0)=\theta_{0}(x), \quad-\infty<x<\infty .
$$

We note that (1.22) implies $\theta_{0}(x)=T_{0}(x)-\bar{T}>0,-\infty<x<\infty$, and so the maximum principle applied to $(2.2)_{3}$ yields

$$
\theta(x, t)>0, \quad-\infty<x<\infty, \quad 0 \leqslant t \leqslant L .
$$

Throughout this section, $\Lambda$ will denote a generic positive constant which may depend at most upon the material constants $\mu, c, \kappa$, on the constants $H, J$ in (1.23), (1.26), and on bounds of $p(u), p_{u}(u), p_{u u}(u)$ on a fixed neighborhood of $\bar{u}$ in which the range of $u(x, t)$ will be confined.

To get the first estimate, we integrate $(1.12)_{3}$, over $(-\infty, \infty) \times[0, t], t \in[0, L]$, and use (1.18), (1.19), (2.1), and (1.25) to conclude

$$
\begin{aligned}
\int_{-\infty}^{\infty}\left\{P(u(x, t))+c \theta(x, t)+\frac{1}{2} v^{2}(x, t)\right\} d x \\
=\int_{-\infty}^{\infty}\left\{P\left(u_{0}(x)\right)+c \theta_{0}(x)+\frac{1}{2} v_{0}^{2}(x)\right\} d x<\tilde{\Lambda} \delta .
\end{aligned}
$$


By virtue of (2.5), the standard theory of parabolic equations (cf. Ladyzenskaja, Solonnikov, and Ural'ceva [9]) for $(2.2)_{3}$ yields that $\theta(x, t)$ is a priori bounded on $(-\infty, \infty) \times[0, L]$. Nevertheless, since we need here an explicit bound on $\max \theta(x, t)$ in terms of $\delta$, we proceed to derive the appropriate estimate by the method of Alikakos [1]. LEMMA 2.1. For any $-\infty<x<\infty, 0 \leqslant t \leqslant L$,

$$
0<\theta(x, t) \leqslant \Lambda \delta \text {. }
$$

Proof. We define

$$
A_{k}=\max _{[0, L]} \int_{-\infty}^{\infty} \theta^{2^{k}}(x, t) d x, \quad k=0,1,2, \ldots,
$$

and proceed to show that

$$
A_{k} \leqslant(\Lambda \delta)^{2^{k}}, \quad k=0,1,2, \ldots
$$

Once (2.8) has been established, the assertion of the lemma will follow by taking the $\left(2^{-k}\right)$ th power and then passing to the limit, $k \rightarrow \infty$.

We fix $k=1,2, \ldots$, multiply $(2.2)_{3}$ by $2^{k} \theta^{2^{k}-1}$, and integrate by parts over $(-\infty, \infty)$ to get

$$
\frac{d}{d t} \int_{-\infty}^{\infty} \theta^{2^{k}} d x=-4\left(1-2^{-k}\right) \frac{\kappa}{c} \int_{-\infty}^{\infty}\left(\partial_{x} \theta^{2^{k-1}}\right)^{2} d x-2 \cdot 2^{k} \frac{\mu}{c} \int_{-\infty}^{\infty}\left(\partial_{x} \theta^{2^{k-1}}\right) \theta^{2^{k-1}} v d x
$$

Using the Cauchy-Schwarz inequality, (2.9) yields

$$
\begin{aligned}
\frac{d}{d t} \int_{-\infty}^{\infty} \theta^{2^{k}} d x & \leqslant-\frac{\kappa}{c} \int_{-\infty}^{\infty}\left(\partial_{x} \theta^{2^{k-1}}\right)^{2} d x+2^{2 k} \frac{\mu^{2}}{\kappa c} \int_{-\infty}^{\infty} \theta^{2^{k}} v^{2} d x \\
& \leqslant-\frac{\kappa}{c} \int_{-\infty}^{\infty}\left(\partial_{x} \theta^{2^{k-1}}\right)^{2} d x+2^{2 k} \frac{\mu^{2}}{\kappa c} \max \theta^{2^{k}} \int_{-\infty}^{\infty} v^{2} d x
\end{aligned}
$$

At the same time, for any $\nu>0$,

$$
\begin{aligned}
\max \theta^{2^{k}} & \leqslant 2 \int_{-\infty}^{\infty} \theta^{2^{k-1}}\left|\partial_{x} \theta^{2^{k-1}}\right| d x \\
& \leqslant \nu \int_{-\infty}^{\infty}\left(\partial_{x} \theta^{2^{k-1}}\right)^{2} d x+\nu^{-1} \int_{-\infty}^{\infty} \theta^{2^{k}} d x \\
& \leqslant \nu \int_{-\infty}^{\infty}\left(\partial_{x} \theta^{2^{k-1}}\right)^{2} d x+\nu^{-1} \max \theta^{2^{k-1}} \int_{-\infty}^{\infty} \theta^{2^{k-1}} d x \\
& \leqslant \nu \int_{-\infty}^{\infty}\left(\partial_{x} \theta^{2^{k-1}}\right)^{2} d x+\frac{1}{2} \max \theta^{2^{k}}+\frac{\nu^{-2}}{2}\left\{\int_{-\infty}^{\infty} \theta^{2^{k-1}} d x\right\}^{2} .
\end{aligned}
$$

Using (2.5) and combining (2.10) with the (Gagliardo-Nirenberg type) inequality (2.11), for $\nu$ appropriately small, yields an estimate of the form

$$
\frac{d}{d t} \int_{-\infty}^{\infty} \theta^{2^{k}} d x \leqslant 2^{6 k} \Lambda\left\{\int_{-\infty}^{\infty} \theta^{2^{k-1}} d x\right\}^{2}
$$


Integrating (2.12), recalling (2.7) and (1.24), (1.25), we deduce

$$
A_{k} \leqslant \delta^{2^{k}}+2^{6 k} \bar{\Lambda} A_{k-1}^{2} \text {. }
$$

We now pick $\Lambda$, with $\Lambda^{1 / 2} \geqslant \max \left\{8,2^{13} \bar{\Lambda}, \tilde{\Lambda}\right\}, \tilde{\Lambda}$ being the constant in (2.5), and claim that

$$
A_{k} \leqslant 2^{-6 k} \Lambda^{-1 / 2}(\Lambda \delta)^{2^{k}}, \quad k=0,1,2, \ldots
$$

Indeed, (2.14) holds for $k=0$, by virtue of (2.5). The validity for any $k$ then follows easily by induction with the help of (2.13). In particular, (2.8) holds. This completes the proof.

The next step is to obtain pointwise bounds for $u(x, t)$ and $v(x, t)$. As in the hyperbolic case, we introduce by (1.7) the Riemann invariants $r, s$ and apply on them the differentiation operators ', ', defined by (1.8). Using $(2.2)_{1}$ and $(2.2)_{2}$, the calculation yields

$$
\grave{r}=\mu \partial_{x} \theta, \quad \dot{s}=\mu \partial_{x} \theta,
$$

which should be compared with (1.9).

In order to handle the right-hand side of (2.15), let us define

$$
\begin{aligned}
\chi(x, t) & =\int_{-\infty}^{x}\left\{\varepsilon(y, t)+\frac{1}{2} v^{2}(y, t)\right\} d y \\
& =\int_{-\infty}^{x}\left\{P(u(y, t))+c \theta(y, t)+\frac{1}{2} v^{2}(y, t)\right\} d y .
\end{aligned}
$$

By virtue of (2.5), (2.4), and (1.19),

$$
0 \leqslant \chi(x, t) \leqslant \tilde{\Lambda} \delta, \quad-\infty<x<\infty, 0 \leqslant t \leqslant L .
$$

Integrating (1.12) $)_{3}$ with respect to $x$ over $(-\infty, x)$ and using (2.16), (2.1), and (1.18) yields

$$
\begin{aligned}
\kappa \partial_{x} \theta & =\partial_{t} \chi-\sigma v \\
& =\dot{\chi}+p_{u}^{1 / 2}\left(P+c \theta+\frac{1}{2} v^{2}\right)-(p+\mu \theta) v \\
& =\dot{\chi}-p_{u}^{1 / 2}\left(P+c \theta+\frac{1}{2} v^{2}\right)-(p+\mu \theta) v .
\end{aligned}
$$

Therefore, setting

$$
\left\{\begin{array}{l}
\phi=r-(\mu / \kappa) \chi \\
\psi=s-(\mu / \kappa) \chi
\end{array}\right.
$$

Eq. (2.15) takes the form

$$
\left\{\begin{array}{l}
\dot{\phi}=(\mu / \kappa) p_{u}^{1 / 2}\left(P+c \theta+\frac{1}{2} v^{2}\right)-(\mu / \kappa)(p+\mu \theta) v, \\
\dot{\psi}=-(\mu / \kappa) p_{u}^{1 / 2}\left(P+c \theta+\frac{1}{2} v^{2}\right)-(\mu / \kappa)(p+\mu \theta) v .
\end{array}\right.
$$

The right-hand side of (2.20) may be expressed in terms of $\phi$ and $\psi$ with the help of

$$
u=E^{-1}(\phi-\psi), \quad v=\frac{1}{2}(\phi+\psi)+(\mu / \kappa) \chi,
$$

where $E$ is the monotone increasing function defined by

$$
E(u)=2 \int_{\bar{u}}^{u} p_{u}^{1 / 2}(\xi) d \xi .
$$


LEMMA 2.2. For any $\eta>0$ there is $\delta>0$, depending on $\eta$, such that when (1.24), (1.25) hold then

$$
|u(x, t)-\bar{u}|<\eta, \quad|v(x, t)|<\eta,
$$

for all $-\infty<x<\infty, 0 \leqslant t \leqslant L$.

Proof. By virtue of (2.21), (2.22), and (2.17), it suffices to show that given any $\bar{\eta}>0$ there is $\delta>0$ such that (1.24), (1.25) imply

$$
|\phi(x, t)|<\bar{\eta}, \quad|\psi(x, t)|<\bar{\eta}, \quad-\infty<x<\infty, 0 \leqslant t \leqslant L .
$$

To this end, let us define, for $t \in[0, L]$,

$$
\Phi(t)=\sup _{x}|\phi(x, t)|, \quad \Psi(t)=\sup _{x}|\psi(x, t)| .
$$

Note that $\Phi$ and $\Psi$ are Lipschitz continuous on $[0, L]$.

We now fix any $t \in(0, L]$ and identify points $\hat{x}$ and $\check{x}$ in $(-\infty, \infty]$ such that

$$
\Phi(t)=|\phi(\hat{x}, t)|, \quad \Psi(t)=|\psi(\check{x}, t)| .
$$

For any $\Delta t \in(0, t]$,

$$
\left\{\begin{array}{l}
\Phi(t-\Delta t) \geqslant\left|\phi\left(\hat{x}+\Delta t p_{u}^{1 / 2}(u(\hat{x}, t)), t-\Delta t\right)\right|, \\
\Psi(t-\Delta t) \geqslant\left|\psi\left(\check{x}-\Delta t p_{u}^{1 / 2}(u(\check{x}, t)), t-\Delta t\right)\right| .
\end{array}\right.
$$

Subtracting (2.27) from (2.26), dividing through by $\Delta t$, and passing to the limit as $\Delta t \downarrow 0$, we deduce

$$
D^{-} \Phi(t) \leqslant|\grave{\phi}(\hat{x}, t)|, \quad D^{-} \Psi(t) \leqslant|\dot{\psi}(\check{x}, t)| .
$$

On the other hand, on account of (1.19), (2.21), (2.22), (2.6), and (2.17), we derive easily the estimate

$$
\left| \pm \frac{\mu}{\kappa} p_{u}^{1 / 2}\left(P+c \theta+\frac{1}{2} v^{2}\right)-\frac{\mu}{\kappa}(p+\mu \theta) v\right| \leqslant \Lambda\left\{[|\phi|+|\psi|]^{2}+[|\phi|+|\psi|]+\delta\right\} .
$$

Combining (2.28), (2.20), (2.25), and (2.29), we conclude that

$$
(d / d t)[\Phi(t)+\Psi(t)] \leqslant \Lambda\left\{[\Phi(t)+\Psi(t)]^{2}+[\Phi(t)+\Psi(t)]+\delta\right\}
$$

for almost all $t \in[0, L]$. At the same time, it follows from (2.25), (2.19), (2.17), (1.7), and (1.24) that

$$
\Phi(0) \leqslant \Lambda \delta^{1 / 2}, \quad \Psi(0) \leqslant \Lambda \delta^{1 / 2} .
$$

Therefore, when $\delta$ is sufficiently small, (2.30), (2.31) imply

$$
\Phi(t)+\Psi(t)<\bar{\eta}, \quad 0 \leqslant t \leqslant L,
$$

whence (2.24) follows. The proof is complete.

We now proceed to estimate the partial derivatives $w, \omega$ of the Riemann invariants defined by (1.10). From (1.7) it follows that

$$
\partial_{x} u=\frac{1}{2} p_{u}^{-1 / 2}(w-\omega), \quad \partial_{x} v=\frac{1}{2}(w+\omega),
$$


whence we easily obtain, using (1.8), (2.2) $)_{1}$,

$$
\grave{u}=\omega, \quad \dot{u}=w .
$$

Taking the partial derivative with respect to $x$ of Eqs. (2.15) yields

$$
\left\{\begin{array}{l}
\partial_{t} \partial_{x} r-p_{u}^{1 / 2} \partial_{x}^{2} r-\frac{1}{2} p_{u}^{-1 / 2} p_{u u} \partial_{x} u \partial_{x} r=\mu \partial_{x}^{2} \theta, \\
\partial_{t} \partial_{x} s+p_{u}^{1 / 2} \partial_{x}^{2} s+\frac{1}{2} p_{u}^{-1 / 2} p_{u u} \partial_{x} u \partial_{x} s=\mu \partial_{x}^{2} \theta .
\end{array}\right.
$$

We substitute $\partial_{x} u$ from (2.33) in (2.35) and then use (1.10), (1.8), and (2.34) to get

$$
\left\{\begin{array}{l}
\grave{w}+\frac{1}{4} p_{u}^{-1} p_{u u} \grave{u} w-\frac{1}{4} p_{u}^{-1} p_{u u} w^{2}=\mu \partial_{x}^{2} \theta \\
\dot{\omega}+\frac{1}{4} p_{u}^{-1} p_{u u} \dot{u} \omega-\frac{1}{4} p_{u}^{-1} p_{u u} \omega^{2}=\mu \partial_{x}^{2} \theta
\end{array}\right.
$$

Multiplying (2.36) by the integrating factor $p_{u}^{1 / 4}$, we obtain

$$
\left\{\begin{array}{l}
\left(p_{u}^{1 / 4} w\right)-\frac{1}{4} p_{u}^{-5 / 4} p_{u u}\left(p_{u}^{1 / 4} w\right)^{2}=\mu p_{u}^{1 / 4} \partial_{x}^{2} \theta \\
\left(p_{u}^{1 / 4} \omega\right)^{\prime}-\frac{1}{4} p_{u}^{-5 / 4} p_{u u}\left(p_{u}^{1 / 4} \omega\right)^{2}=\mu p_{u}^{1 / 4} \partial_{x}^{2} \theta
\end{array}\right.
$$

which is the analog of Eqs. (1.11).

In order to handle the right-hand side of (2.37), we combine (2.2) 3 with (2.33) and (1.8), thus obtaining

$$
\begin{aligned}
\partial_{x}^{2} \theta & =\frac{c}{\kappa} \partial_{t} \theta-\frac{\mu}{\kappa} \theta \partial_{x} v \\
& =\frac{c}{\kappa} \dot{\theta}+\frac{c}{\kappa} p_{u}^{1 / 2} \partial_{x} \theta-\frac{\mu}{2 \kappa} \theta(w+\omega) \\
& =\frac{c}{\kappa} \dot{\theta}-\frac{c}{\kappa} p_{u}^{1 / 2} \partial_{x} \theta-\frac{\mu}{2 \kappa} \theta(w+\omega) .
\end{aligned}
$$

At the same time, on account of (2.34),

$$
\left\{\begin{array}{l}
p_{u}^{1 / 4} \dot{\theta}=\left(p_{u}^{1 / 4} \theta\right)^{\prime}-\frac{1}{4} p_{u}^{-3 / 4} p_{u u} \omega \theta \\
p_{u}^{1 / 4} \dot{\theta}=\left(p_{u}^{1 / 4} \theta\right)^{\prime}-\frac{1}{4} p_{u}^{-3 / 4} p_{u u} w \theta
\end{array}\right.
$$

Therefore, setting

$$
f=p_{u}^{1 / 4}\left(w-\frac{\mu c}{\kappa} \theta\right), \quad g=p_{u}^{1 / 4}\left(\omega-\frac{\mu c}{\kappa} \theta\right)
$$

we may write (2.37) in the form

$$
\begin{aligned}
\grave{f}= & \frac{1}{4} p_{u}^{-5 / 4} p_{u u} f^{2}+\frac{\mu c}{4 \kappa} p_{u}^{-1} p_{u u} \theta(2 f-g)-\frac{\mu^{2}}{2 \kappa} \theta(f+g) \\
& +\frac{\mu c}{\kappa} p_{u}^{3 / 4} \partial_{x} \theta-\frac{\mu^{3} c}{\kappa^{2}} p_{u}^{1 / 4} \theta^{2} \\
\dot{g}= & \frac{1}{4} p_{u}^{-5 / 4} p_{u u} g^{2}+\frac{\mu c}{4 \kappa} p_{u}^{-1} p_{u u} \theta(2 g-f)-\frac{\mu^{2}}{2 \kappa} \theta(f+g) \\
& -\frac{\mu c}{\kappa} p_{u}^{3 / 4} \partial_{x} \theta-\frac{\mu^{3} c}{\kappa^{2}} p_{u}^{1 / 4} \theta^{2} .
\end{aligned}
$$


In order to estimate the term that contains $\partial_{x} \theta$ in (2.41), we define the Lipschitz continuous functions $F, G$ on $[0, L]$ by

$$
F(t)=\max _{x}|f(x, t)|, \quad G(t)=\max _{x}|g(x, t)|
$$

and state

LEMMA 2.3. For any $-\infty<x<\infty, 0 \leqslant t \leqslant L$,

$$
\left|\partial_{x} \theta(x, t)\right| \leqslant \Lambda+\Lambda \delta \int_{0}^{t} \frac{1}{\sqrt{t-\tau}}[F(\tau)+G(\tau)] d \tau .
$$

Proof. By virtue of $(2.2)_{3}, \theta$ admits the representation

$$
\begin{aligned}
\boldsymbol{\theta}(x, t)= & \left(\frac{c}{4 \pi \kappa t}\right)^{1 / 2} \int_{-\infty}^{\infty} \exp \left\{-\frac{c}{4 \kappa} \frac{(x-y)^{2}}{t}\right\} \theta_{0}(y) d y \\
& +\left(\frac{\mu^{2}}{4 \pi \kappa c}\right)^{1 / 2} \int_{0}^{t} \int_{-\infty}^{\infty}(t-\tau)^{-1 / 2} \exp \left\{-\frac{c}{4 \kappa} \frac{(x-y)^{2}}{t-\tau}\right\} \theta(y, \tau) \partial_{x} v(y, \tau) d y d \tau .
\end{aligned}
$$

Using (2.33) and (2.40), we substitute $\partial_{x} v$ in (2.44) in terms of $f, g$, and $\theta$. Differentiating the resulting equation with respect to $t$, we derive the following representation for $\partial_{x} \theta$ :

$$
\begin{aligned}
\partial_{x} \theta(x, t)= & \left(\frac{c}{4 \pi \kappa t}\right)^{1 / 2} \int_{-\infty}^{\infty} \exp \left\{-\frac{c}{4 \kappa} \frac{(x-y)^{2}}{t}\right\} \partial_{x} \theta_{0} d y \\
& -\left(\frac{\mu^{2} c}{64 \pi \kappa^{3}}\right)^{1 / 2} \int_{0}^{t} \int_{-\infty}^{\infty} \frac{x-y}{(t-\tau)^{3 / 2}} \exp \left\{-\frac{c}{4 \kappa} \frac{(x-y)^{2}}{t-\tau}\right\} \\
& \cdot \theta\left\{p_{u}^{-1 / 4}(f+g)+\frac{2 \mu c}{\kappa} \theta\right\} d y d \tau .
\end{aligned}
$$

Estimate (2.43) now follows easily from (2.45) with the help of (1.23), (2.6), and (2.42). This completes the proof.

We have now laid the preparation for proving Theorem 1.1. The idea of the proof is, of course, that the quadratic terms in (2.41) induce blow up of $f$ and/or $g$ in finite time.

Proof of Theorem 1.1. Let us define nonnegative Lipschitz continuous functions $F^{+}, G^{+}$, $F^{-}, G^{-}$on $[0, L]$ by

$$
\begin{array}{cc}
F^{+}(t)=\sup _{x} f(x, t), & G^{+}(t)=\sup _{x} g(x, t), \\
F^{-}(t)=-\inf _{x} f(x, t), & G^{-}(t)=-\inf _{x} g(x, t) .
\end{array}
$$

It is clear that, for $t \in[0, L]$,

$$
F(t) \leqslant F^{+}(t)+F^{-}(t), \quad G(t) \leqslant G^{+}(t)+G^{-}(t) .
$$

We fix any $t \in[0, L)$ with $F^{+}(t)>0$ and/or $G^{+}(t)>0$ and identify points $\hat{x}$ and $\check{x}$ in $(-\infty, \infty)$ such that

$$
F^{+}(t)=f(\hat{x}, t) \text { and } / \text { or } \quad G^{+}(t)=g(\check{x}, t) .
$$


For any $\Delta t \in(0, L-t]$,

$$
\left\{\begin{array}{l}
F^{+}(t+\Delta t) \geqslant f\left(\hat{x}-\Delta t p_{u}^{1 / 2}(u(\hat{x}, t)), t+\Delta t\right), \\
G^{+}(t+\Delta t) \geqslant g\left(\check{x}+\Delta t p_{u}^{1 / 2}(u(\check{x}, t)), t+\Delta t\right) .
\end{array}\right.
$$

Subtracting (2.49) from (2.50), dividing through by $\Delta t$, and passing to the limit as $\Delta t \downarrow 0$, we obtain

$$
D^{+} F^{+}(t) \geqslant \grave{f}(\hat{x}, t) \text { and /or } D^{+} G^{+}(t) \geqslant \dot{g}(\check{x}, t) .
$$

Next we fix any $t \in(0, L]$ with $F^{-}(t)>0$ and/or $G^{-}(t)>0$ and identify points $\hat{y}$ and /or $\check{y}$ in $(-\infty, \infty)$ such that

$$
F^{-}(t)=-f(\hat{y}, t) \quad \text { and } / \text { or } \quad G^{-}(t)=-g(\check{y}, t) .
$$

For any $\Delta t \in(0, t]$,

$$
\left\{\begin{array}{l}
F^{-}(t-\Delta t) \geqslant-f\left(\hat{y}+\Delta t p_{u}^{1 / 2}(u(\hat{y}, t)), t-\Delta t\right), \\
G^{-}(t-\Delta t) \geqslant-g\left(\check{y}-\Delta t p_{u}^{1 / 2}(u(\check{y}, t)), t-\Delta t\right) .
\end{array}\right.
$$

We subtract (2.53) from (2.52), then we divide through by $\Delta t$, and pass to the limit as $\Delta t \downarrow 0$, thus obtaining

$$
D^{-} F^{-}(t) \leqslant-\dot{f}(\hat{y}, t) \text { and/or } D^{-} G^{-}(t) \leqslant-\dot{g}(\check{y}, t) .
$$

In view of our assumption (1.20) and Lemma 2.2, there is a positive constant $\alpha$ such that for all $-\infty<x<\infty, 0 \leqslant t \leqslant L$,

$$
\frac{1}{4} p_{u}^{-5 / 4}(u(x, t)) p_{u u}(u(x, t)) \geqslant 2 \alpha>0 .
$$

Therefore, combining (2.51) with (2.41) and using (2.55), (2.6), (2.42), (2.48), (2.43), we end up with an estimate of the form

$$
\begin{aligned}
(d / d t)\left[F^{+}(t)+G^{+}(g)\right] \geqslant & \alpha\left[F^{+}(t)+G^{+}(t)\right]^{2}-\Lambda \delta\left[F^{+}(t)+G^{+}(t)\right] \\
& -\Lambda \delta\left[F^{-}(t)+G^{-}(t)\right] \\
& -\Lambda \delta \int_{0}^{t} \frac{1}{\sqrt{t-\tau}}\left[F^{+}(\tau)+G^{+}(\tau)\right] d \tau \\
& -\Lambda \delta \int_{0}^{t} \frac{1}{\sqrt{t-\tau}}\left[F^{-}(\tau)+G^{-}(\tau)\right] d \tau-\Lambda,
\end{aligned}
$$

for almost all $t \in[0, L]$. Similarly, from (2.54), (2.41), (2.55), (2.6), (2.42), (2.48), and (2.43), we obtain, for almost all $t \in[0, L]$,

$$
\begin{aligned}
& (d / d t)\left[F^{-}(t)+G^{-}(t)\right] \leqslant \Lambda \delta\left[F^{+}(t)+G^{+}(t)\right]+\Lambda \delta\left[F^{-}(t)+G^{-}(t)\right] \\
& \quad+\Lambda \delta \int_{0}^{t} \frac{1}{\sqrt{t-\tau}}\left[F^{+}(\tau)+G^{+}(\tau)\right] d \tau+\Lambda \delta \int_{0}^{t} \frac{1}{\sqrt{t-\tau}}\left[F^{-}(\tau)+G^{-}(\tau)\right] d \tau+\Lambda .
\end{aligned}
$$

Upon using (2.47), (2.40), (2.6), (1.26), and Lemma 2.2, it follows from (2.57)

$$
\left[F^{-}(t)+G^{-}(t)\right] \leqslant \Lambda+\Lambda \delta \int_{0}^{t} K(t-\tau)\left[F^{+}(\tau)+G^{+}(\tau)\right] d \tau
$$


where $K$ is a bounded kernel on $[0, L]$. Combining (2.56) with (2.58) we end up with an estimate

$$
\begin{aligned}
(d / d t)\left[F^{+}(t)+G^{+}(t)\right] \geqslant & \alpha\left[F^{+}(t)+G^{+}(t)\right]^{2}-\Lambda \delta\left[F^{+}(t)+G^{+}(t)\right] \\
& -\Lambda \delta \int_{0}^{t} Z(t-\tau)\left[F^{+}(\tau)+G^{+}(\tau)\right] d \tau-\Lambda,
\end{aligned}
$$

where $Z \in L^{1}(0, L)$. Recalling (2.46), (2.40), (2.6), and Lemma 2.2, we now conclude from (2.60) that when (1.27) holds with $M$ sufficiently large, then $F^{+}(t)+G^{+}(t)$ will blow up in a finite time, not exceeding $L$. This completes the proof.

\section{REFERENCES}

[1] N. D. Alikakos, An application of the invariance principle to reaction-diffusion equations, J. Differential Equations 33, 201-225 (1979)

[2] B. D. Coleman, and M. E. Gurtin, Waves in materials with memory III. Thermodynamic influences on the growth and decay of acceleration waves, Arch. Rat. Mech. Anal. 19, 266-298 (1965)

[3] C. M. Dafermos, Contemporary Issues in the Dynamic Behavior of Continuous Media, LCDS Lecture Notes No. $85-1$

[4] C. M. Dafermos, Development of singularities in the motion of materials with fading memory, Arch. Rat. Mech. Anal. 91, 193-205 (1985)

[5] C. M. Dafermos and J. A. Nohel, A nonlinear hyperbolic Volterra equation in viscoelasticity, Amer. J. Math. (suppl. dedicated to P. Hartman) 87-116 (1981)

[6] H. Hattori, Breakdown of smooth solutions in dissipative nonlinear hyperbolic equations, Quart. Appl. Math. 40, 113-127 (1982)

[7] W. J. Hrusa, A nonlinear functional differential equation in Banach space with applications to materials with fading memory, Arch. Rat. Mech. Anal. 84, 99-137 (1983)

[8] S. Klainerman, and A. Majda, Formation of singularities for wave equations including the nonlinear vibrating string, Comm. Pure Appl. Math. 33, 241-263 (1980)

[9] O. A. Ladyženskaja, V. A. Solonnikov, and N. N. Ural'ceva, Linear and Quasilinear Equations of Parabolic Type, Transl. Math. Monographs 23 (1968)

[10] P. D. Lax, Development of singularities of solutions of nonlinear hyperbolic partial differential equations, J. Math. Physics 5, 611-613 (1964)

[11] R. C. MacCamy, A model for one-dimensional, nonlinear viscoelasticity, Quart. Appl. Math. 35, 21-33 (1977)

[12] R. S. McIntire, Ph.D. Dissertation, Brown University (1985)

[13] T. Nishida, Global smooth solutions for the second order quasilinear equation with first order dissipation (unpublished)

[14] M. Slemrod, Global existence, uniqueness and asymptotic stability of classical smooth solutions in one-dimensional, non-linear thermoelasticity, Arch. Rat. Mech. Anal. 76, 97-133 (1981) 